\title{
Prevalence of extra-articular manifestations in patients with ankylosing spondylitis: a systematic review and meta-analysis
}

\author{
Carmen Stolwijk, ${ }^{1,2}$ Astrid van Tubergen, ${ }^{1,2}$ José Dionisio Castillo-Ortiz, ${ }^{3}$ \\ Annelies Boonen ${ }^{1,2}$
}

Handling editor Tore K Kvien

- Additional material is published online only. To view please visit the journal online (http://dx.doi.org/10.1136/ annrheumdis-2013-203582)

1 Department of Medicine, Division of Rheumatology, Maastricht University Medical Center, Maastricht, The Netherlands

${ }^{2}$ School for Public Health and Primary Care (CAPHRI), University of Maastricht, Maastricht, The Netherlands ${ }^{3}$ Unidad de Investigacion em Enfermadades CronicoDegenerativas, Hospital General de Zona 50 Guadalajara, Mexico

\section{Correspondence to} Dr Carmen Stolwijk Department of Medicine, Division of Rheumatology, Maastricht University Medical Center; School for Public Health and Primary Care (CAPHRI), University of Maastricht, PO Box 5800, Maastricht $6202 \mathrm{AZ}$, The Netherlands: c.stolwijk@maastrichtuniversity. $\mathrm{nl}$

Received 7 March 2013 Revised 25 June 2013 Accepted 18 August 2013 Published Online First 2 September 2013

\section{ABSTRACT}

Objectives Uveitis, psoriasis and inflammatory bowel disease (IBD) are common extra-articular manifestations (EAM) in patients with ankylosing spondylitis (AS); however, summary data of reported prevalence are lacking. The aim of the present study was to summarise the prevalence of EAMs among patients with AS and to identify underlying factors to explain potential heterogeneity of prevalence.

Methods A systematic literature search was performed (Medline, Embase and Cochrane Library) to identify relevant articles. Risk of bias was assessed and data were extracted. Pooled prevalences were calculated. Potential sources of any observed clinical or methodological heterogeneity in the estimates were explored by subgroup and metaregression analysis.

Results In the 156 selected articles, 143 reported the prevalence of uveitis (44 372 patients), 56 of psoriasis (27 626 patients) and 69 of IBD (30 410 patients). Substantial heterogeneity was observed in prevalence estimates among all EAMs $\left(I^{2}=84-95 \%\right)$. The pooled prevalence of uveitis was $25.8 \%(95 \% \mathrm{Cl} 24.1 \%$ to $27.6 \%$, and was positively associated in multivariable metaregression with disease duration $(\beta 0.05,95 \% \mathrm{Cl}$ 0.03 to 0.08 ) and random selection of patients ( $\beta-0.24,95 \% \mathrm{Cl}-0.43$ to -0.04$)$. The pooled prevalence of psoriasis was $9.3 \%$ (95\% Cl $8.1 \%$ to $10.6 \%)$. The pooled prevalence of IBD was $6.8 \%(95 \%$ $\mathrm{Cl} 6.1 \%$ to $7.7 \%$ ) and was positively associated with the percentage of women in the studies ( $\beta 0.02,95 \%$ $\mathrm{Cl} 0.00$ to 0.03 ). Geographical area was associated in multivariable metaregressions with prevalence of all EAMs.

Conclusions EAMs are common in patients with AS. The large heterogeneity between studies can be partly explained by differences in clinical as well as methodological characteristics.

\section{INTRODUCTION}

Ankylosing spondylitis (AS) is the prototype of a group of diseases referred to as spondyloarthritis (SpA). ${ }^{1}$ This group of diseases shares genetic, radiographic and clinical features including an increased prevalence of acute anterior uveitis (AAU), psoriasis and inflammatory bowel disease (IBD), the so-called extra-articular manifestations (EAM). ${ }^{2}$

The attention to EAMs in AS has increased in the past years. First, the presence of AAU, psoriasis and IBD in patients with (inflammatory) back pain may help to make a diagnosis of axial $\mathrm{SpA}$
$(\operatorname{axSpA}){ }^{3} \mathrm{Next}$, the presence of one or more EAMs can affect the prognosis of AS and may influence health outcomes including quality of life, work outcome and health resource usage. ${ }^{4-7}$ Moreover, EAMs, in patients with AS, may influence treatment decisions. ${ }^{2}$ Last, insight in the coexistence of EAMs in patients with AS might help to unravel the role of genetic and epigenetic phenomena in the pathophysiology of inflammatory processes involved in SpA. ${ }^{8}$

As a consequence, epidemiological data on the prevalence of EAMs have become increasingly important for clinicians and researchers when trying to understand disease patterns. However, although many studies report the occurrence of the EAMs, only one systematic literature review (SLR), providing only descriptive results and no meta-analysis, is currently available on the prevalence of AAU in SpA. ${ }^{9}$ Furthermore, prevalences of the individual EAMs that are reported in available studies vary substantially. It is currently unclear whether factors can be identified which may explain this variation.

The aims of the present study were (1) to summarise and evaluate the published estimates for the prevalence of EAMs among patients with AS, and (2) to explore the effect of various clinical and methodological factors on reported prevalence. We hypothesised that the prevalences of all EAMs would be associated with disease duration and with geographical area, the latter being associated with differences in distribution of HLA-B27 positivity. Further, it was hypothesised, that the prevalence of EAMs would be associated with the method of ascertainment, with higher prevalences in studies based on self-report.

\section{METHODS}

An SLR with meta-analysis and metaregression analysis was performed in order to obtain best possible estimates of the prevalence of AAU, psoriasis and IBD in patients with AS.

\section{Search strategy and selection criteria}

An SLR was conducted in MEDLINE (1 January 1984 to 1 August 2012), EMBASE (1 January 1989 to 1 August 2012) and the Cochrane Central Register of Controlled trials (CENTRAL). The search strategy, developed with assistance of an experienced librarian and adapted for each database (see online supplementary text S1), consisted 
of a combination of free terms and controlled vocabulary terms relating to AS and to the EAMs. Additionally, 'snowballing' was used to augment the search sensitivity, including reference tracking, checking the search results of other SLRs on AS, which were performed by our research team during the same period, and personal knowledge of the authors. There were no restrictions in language of the papers. One author selected potentially relevant articles after reading the title, keywords and abstract. If in doubt, the full article was read. For inclusion, studies had to meet the following criteria: presenting primary data including the prevalence of AAU, psoriasis and/or IBD in patients ( $\geq 16$ years) with a clinical diagnosis of AS. The prevalence of EAMs did not have to be the main objective of the study and as a consequence, also studies in which EAMs were reported in the baseline description of the study population were considered. Studies including patients with other diagnoses besides AS were only eligible if the results from patients with AS were presented separately. Excluded were abstracts not (yet) published as full manuscripts; opinion or discussion papers; case reports and studies including $<20$ patients. If more than one article reported the same cohort of patients, only the first published article was included.

\section{Risk of bias}

Each study was independently rated by two reviewers for risk of bias based on the Methodological Evaluation of Observational research (MORE) checklist, which was adapted to the specific research question. ${ }^{10}$ External validity is defined as the extent to which the results of a study can be generalised to the target population and comprised, for the present study, the following questions: (1) Was the sampling frame a close representation of the target population? (2) Was an appropriate case definition for AS used? (3) Was some form of random selection used to select the sample? and (4) Was the likelihood of non-response bias minimal?. The internal validity is defined as the possible amount of error in measuring the conditions, and included the following question: (5) Was an acceptable case definition for the EAM used?. ${ }^{10}$ For each criterion, three options were possible: '+'=low risk of bias, '-'=possible risk of bias or '?'= risk of bias unclear due to poor reporting. The predefined rating procedure is shown in the online supplementary table S1. In case of any discrepancies between the two reviewers, a third reviewer was consulted for final decision. For each criterion a score of ' 1 ' (low risk of bias) or ' 0 ' (possible or unclear risk of bias) was given. Finally, a composite score was calculated as the sum of the score on the individual criteria. The criterion 'response rate' was not considered in the composite score because too many scores were not reported.

\section{Data extraction}

Data extraction of the first 20 articles was performed by two reviewers using a standardised data extraction form. Because the agreement on this data extraction was $100 \%$, the remaining of the data extraction was performed by only one reviewer. Data extraction included (if available): study identification (first author, journal, year of publication), study characteristics (study design, geographical area, sample size), patient characteristics (average age of patients, percentage of women, percentage of HLA-B27-positive patients, mean disease duration of AS (years since diagnosis)), and study results (number of patients with (a history of) AAU, psoriasis or IBD). A native speaker or translator was asked for help with articles published in languages other than English, Dutch, Spanish or Portuguese.

\section{Statistical analysis}

The pooled prevalence with 95\% CI of each EAM was estimated using inverse variance weighting. The heterogeneity among studies for each EAM was tested by Cochran's Q test and the $\mathrm{I}^{2}$ statistic, the latter describing the percentage of variation across studies. ${ }^{11}$ Whenever heterogeneity was high $\left(\mathrm{I}^{2}>50 \%\right)$, random-effects models were used. ${ }^{12}$ For all analyses, logit transformation was applied to the prevalence estimates to improve their statistical properties. ${ }^{13}$ The final pooled logit results and 95\% CIs were back-transformed to prevalence estimates for ease of interpretation. Potential sources of heterogeneity were first investigated by an exploratory subgroup analysis, using random effects analogue to one-way analysis of variance, in which groups of studies were arranged according to potentially relevant clinical characteristics (disease duration; percentage of women; percentage of HLA-B27-positive patients; and geographical area, all subdivided in quartiles) and methodological characteristics (study design; sample size; sampling frame; case definition for AS; random selection of patients; response rate; case definition for EAM; and the composite score for risk of bias). Second, random-effects meta-regression analysis via iterative maximum likelihood was performed to explore associations between the clinical and methodological characteristics (moderators) and the outcome. ${ }^{14}$ The advantage of metaregression analysis, compared to subgroup analysis, is the possibility of evaluation of continuous moderators and evaluation of more than one moderator simultaneously. In the metaregression analysis, the same clinical characteristics were entered as in the subgroup analysis, but now as continuous variables, as appropriate. The influence of methodological characteristics was evaluated by the individual risk of bias criteria (low risk vs possible risk of bias) as well as study design and sample size.

Variables with a $p$ value of $<0.30$ in the univariable analysis were entered in the multivariable model. Multicollinearity between variables was checked by computing variance inflation factors (VIF) before inclusion into the model. ${ }^{15} \mathrm{~A}$ backward procedure was used, removing variables with a $\mathrm{p}$ value of $>0.05$ in the multivariable model in order of significance, until the best-fitting model was identified. For categorical variables, the $\mathrm{p}$ value was calculated for all dummy variables together $\left(\mathrm{R}^{2}\right.$ change). Interactions between the variables were explored by additional analyses that included the main effect variables and the interaction term for each interaction studied. All statistical analyses were conducted using SPSS V.18.0. For the metaregression analysis, SPSS macros were used (Metareg.sps and MetaF.sps). ${ }^{16}$

\section{RESULTS \\ Search}

The search in databases resulted in 5898 articles (figure 1). After excluding duplicate articles and screening on title and abstract, 385 articles remained for full-paper review. Three fulltext articles could not be retrieved, despite efforts to contact the corresponding author. After reading the full text of the 382 articles, 94 articles were included. The main reasons for exclusion were no primary data reported or no data on patients with AS. The additional search resulted in inclusion of another 62 articles. In total, 156 articles were included.

\section{Characteristics of the included studies}

The table of individual studies with full references and detailed characteristics is available in the online supplementary table S2. A summary of the clinical characteristics of the included studies 
Figure 1 Flow-diagram of systematic literature search. $\mathrm{n}=$ number of studies.

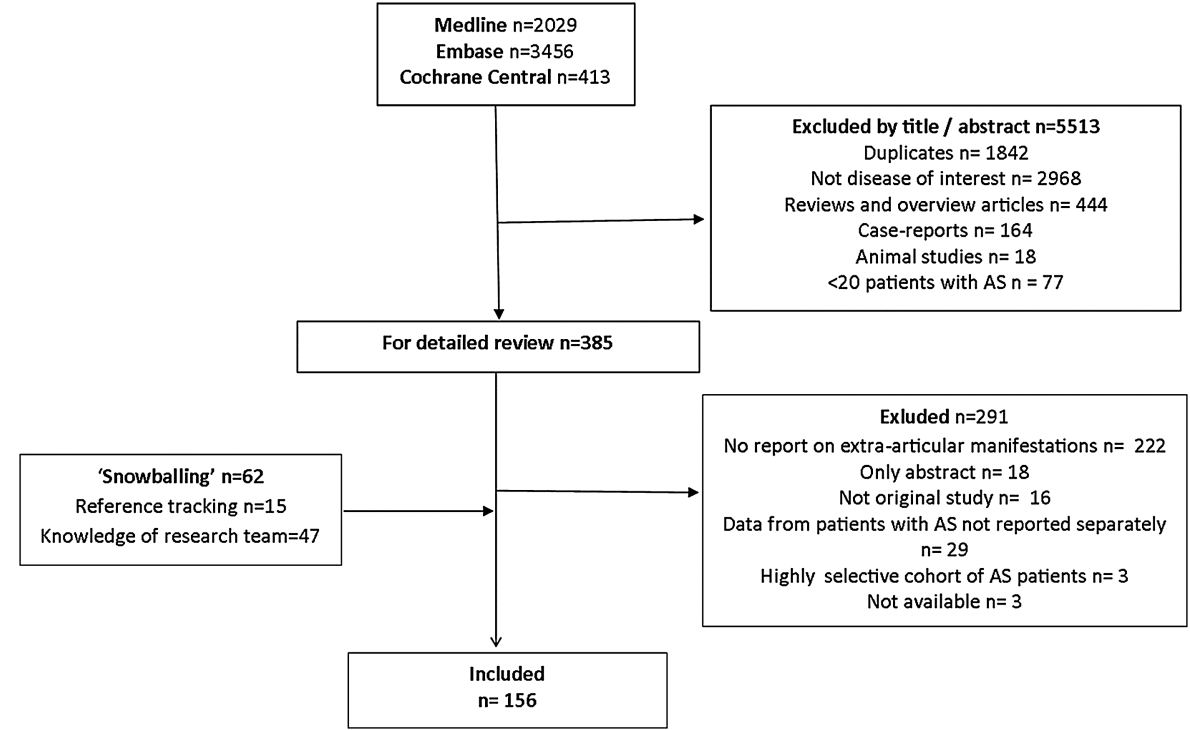

is shown in table 1 . The prevalence of AAU in patients with AS was reported in 143 articles $(85$ cross-sectional, 9 prospective, 28 retrospective, 20 intervention and 1 case-control study) and included 44372 patients of which 13071 (29.5\%) patients had a history of AAU. The prevalence of psoriasis was reported in 56 articles ( 28 cross-sectional, 5 prospective, 9 retrospective, 12 intervention and 2 case-control studies) and included 27626 patients, of which $2980(10.8 \%)$ patients had (a history of) psoriasis. The prevalence of IBD was reported in 69 articles (38 cross-sectional, 7 prospective, 10 retrospective, 12 intervention and 2 case-control studies), and included 32341 patients, of which $2251(7.0 \%)$ patients had (a history of) IBD.

\section{Risk of bias}

Risk of bias was assessed in all 156 studies. A risk of bias summary for all included studies is shown in online supplementary table S2, and a risk of bias graph for each EAM in online supplementary figure $\mathrm{S} 1$, respectively.

With respect to the external validity, the most frequent source of bias was found in the random selection of patients, for which in only $20-26 \%$ of the studies the risk of bias was considered low. The risk of bias due to the sampling frame or the case definition for AS was considered low in $78-87 \%$ and $58-79 \%$ of the studies, respectively. The response rate was reported in 20 $30 \%$ of the studies only, and therefore, the non-response bias was difficult to assess. With respect to internal validity, the risk of bias of the case definition for the EAM was considered low in $43-51 \%$ of the studies.

\section{Meta-analysis and subgroup analysis}

Acute anterior uveitis

The pooled prevalence of AAU was $25.8 \%$ (95\% CI $24.1 \%$ to $27.6 \%)$ with a substantial heterogeneity observed $(Q=2374.63$,

Table 1 Clinical characteristics of the 156 included studies specified for each extra-articular manifestation

\begin{tabular}{|c|c|c|c|c|c|c|c|c|c|}
\hline & \multicolumn{3}{|l|}{ AAU } & \multicolumn{3}{|l|}{ Psoriasis } & \multicolumn{3}{|l|}{ IBD } \\
\hline & $\begin{array}{l}\text { Number of } \\
\text { studies }(\%)^{*}\end{array}$ & $\begin{array}{l}\text { Number of } \\
\text { patients }(\%)^{*}\end{array}$ & $\begin{array}{l}\text { Weighted } \\
\text { mean (SD)† }\end{array}$ & $\begin{array}{l}\text { Number of } \\
\text { studies }(\%)^{*}\end{array}$ & $\begin{array}{l}\text { Number of } \\
\text { patients }(\%)^{*}\end{array}$ & $\begin{array}{l}\text { Weighted } \\
\text { mean (SD)† }\end{array}$ & $\begin{array}{l}\text { Number of } \\
\text { studies }(\%)^{*}\end{array}$ & $\begin{array}{l}\text { Number of } \\
\text { patients }(\%)^{*}\end{array}$ & $\begin{array}{l}\text { Weighted } \\
\text { mean (SD) } \dagger\end{array}$ \\
\hline Total & 143 & 44372 & - & 56 & 27626 & - & 69 & 32341 & - \\
\hline Age in years & $112(78.3)$ & $33650(75.8)$ & $43.1(5.9)$ & $49(87.5)$ & $20267(73.4)$ & $45.3(4.6)$ & $61(88.4)$ & $24836(76.8)$ & $45.0(4.8)$ \\
\hline $\begin{array}{l}\text { Disease duration in } \\
\text { years }\end{array}$ & $119(83.2)$ & $33444(75.4)$ & $15.9(5.9)$ & $50(89.3)$ & $19612(71.0)$ & $16.7(6.2)$ & $60(87.0)$ & $23016(71.2)$ & $16.7(6.3)$ \\
\hline$\%$ Men & $134(93.7)$ & $35767(80.6)$ & $75.5(8.5)$ & $53(94.6)$ & $20821(75.4)$ & $73.0(7.3)$ & $65(94.2)$ & $25488(78.8)$ & $73.3(7.5)$ \\
\hline$\%$ HLA-B27+ & $105(73.4)$ & $27327(61.6)$ & $84.0(11.3)$ & $39(69.6)$ & $16231(58.8)$ & $79.9(11.0)$ & $43(62.3)$ & $18052(55.8)$ & $81.2(10.4)$ \\
\hline \multicolumn{10}{|c|}{ Geographical area of study } \\
\hline Europe & $65(45.5)$ & $25006(56.4)$ & - & $35(62.5)$ & $21740(78.7)$ & - & $39(56.5)$ & $20123(62.2)$ & - \\
\hline North America & $14(9.8)$ & $2901(6.5)$ & - & $10(17.9)$ & 2007 (7.3) & - & $10(14.5)$ & $2866(8.9)$ & - \\
\hline Latin America & $11(7.7)$ & $1358(3.1)$ & - & $1(1.8)$ & $58(0.2)$ & - & $3(4.3)$ & $380(1.2)$ & - \\
\hline Asia & $34(23.8)$ & $9923(22.4)$ & - & $2(3.6)$ & $2057(7.4)$ & - & $6(8.7)$ & $3331(10.3)$ & - \\
\hline Middle East & $14(9.8)$ & $1502(3.4)$ & - & $4(7.1)$ & 537 (1.9) & - & $5(7.2)$ & 503 (1.6) & - \\
\hline Australia & $1(0.7)$ & $74(0.2)$ & - & 0 & 0 & - & 0 & 0 & - \\
\hline Several areas & $4(2.8)$ & $3608(8.1)$ & - & $4(7.1)$ & $1227(4.4)$ & - & $6(8.7)$ & $5138(15.9)$ & - \\
\hline
\end{tabular}




\section{Clinical and epidemiological research}

$\left.\mathrm{p}<0.01, \mathrm{I}^{2}=94.0 \%\right)$. Figure 2 shows the prevalence estimates of AAU according to the different clinical and methodological characteristics. The prevalence of AAU was significantly associated with disease duration, and increased from 17.4\% (95\% CI $14.3 \%$ to $21.3 \%$ ) in studies including patients with a mean disease duration of $<10$ years to $38.5 \%$ (95\% CI $33.3 \%$ to
$43.9 \%$ ) in those with a mean disease duration of $>20$ years. Furthermore, the prevalence was significantly different across geographical regions. Prevalence estimates were highest in studies from North America (35.2\%) and Europe (29.3\%), and lower in studies from Asia (21.4\%) and Latin America (20.1\%). The prevalence of AAU also varied significantly, according to

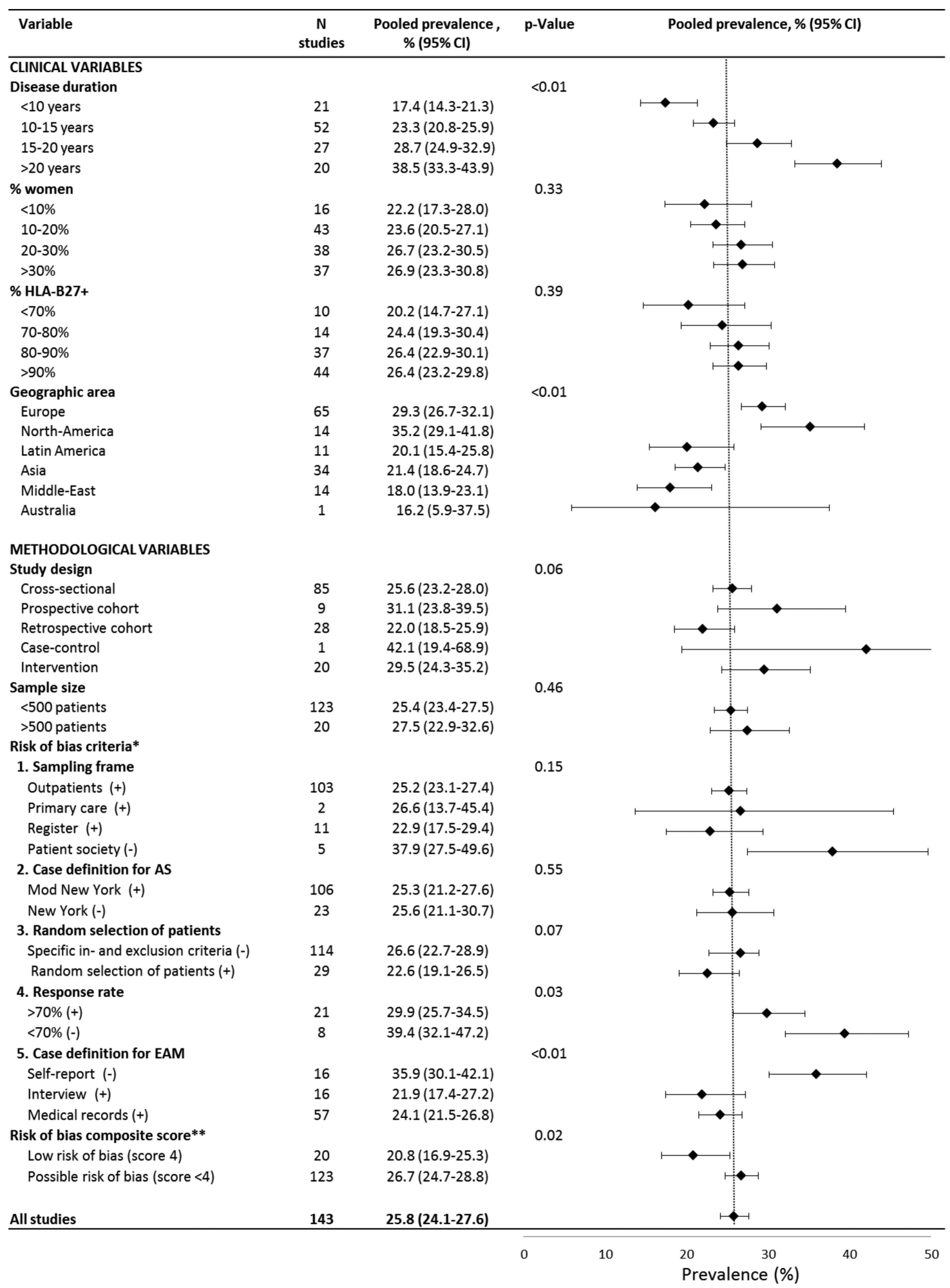

Figure 2 Prevalence of acute anterior uveitis in patients with ankylosing spondylitis grouped by clinical and methodological characteristics. EAM, extra-articular manifestation; * Individual risk of bias criteria, ' + ' considered as low risk of bias, ' - ' as high risk of bias. ${ }^{* *}$ Sum of individual risk of bias scores, the item on response rate was not considered in the composite score because too many scores were not reported. 
the case definition for the EAM, and was highest for self-report (35.9\%). Finally, the prevalence was lower in studies considered to have a 'low risk of bias' $(20.8 \%, 95 \%$ CI $16.9 \%$ to $25.3 \%)$ compared with studies with a high risk of bias $(26.7 \%, 95 \%$ CI $24.7 \%$ to $28.8 \%)$.

\section{Psoriasis}

The pooled prevalence of psoriasis was $9.3 \%$ (95\% CI $8.1 \%$ to $10.6 \%)$ with substantial heterogeneity observed $(\mathrm{Q}=608.17$, $\left.\mathrm{p}<0.01, \mathrm{I}^{2}=91.0 \%\right)$. Figure 3 shows the prevalence of psoriasis according to the different clinical and methodological characteristics. The reported prevalence of psoriasis varied significantly between different geographical areas, with the highest prevalence in studies from Europe (10.9\%), and the lowest in studies from Asia (3.1\%) and the Middle East (4.2\%).

\section{Inflammatory bowel disease}

The pooled prevalence of IBD was $6.8 \%$ (95\% CI 6.1\% to $7.7 \%)$ with a substantial heterogeneity observed $(\mathrm{Q}=422.80, \mathrm{p}<0.01$, $\mathrm{I}^{2}=84.2 \%$ ). Figure 4 shows the prevalence of IBD according to

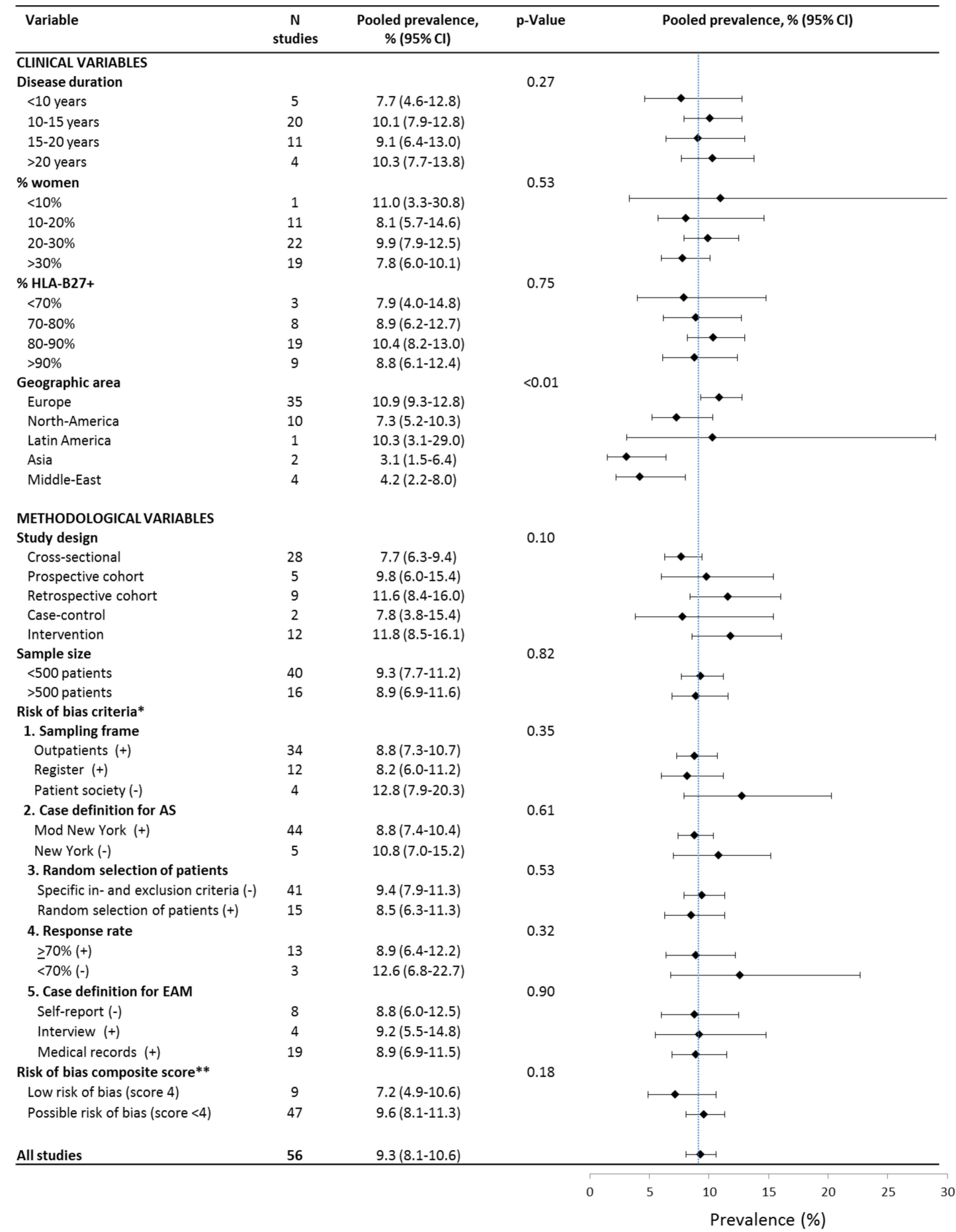

Figure 3 Prevalence of psoriasis in patients with ankylosing spondylitis grouped by clinical and methodological characteristics. EAM, extra-articular manifestation; * Individual risk of bias criteria, ' +' considered as low risk of bias, '-' as high risk of bias. **Sum of individual risk of bias scores, the item on response rate was not considered in the composite score because too many scores were not reported. 


\begin{tabular}{|c|c|c|c|c|}
\hline & $\begin{array}{c}\mathrm{N} \\
\text { studies }\end{array}$ & $\begin{array}{l}\text { Pooled prevalence } \\
\%,(95 \% \mathrm{Cl})\end{array}$ & P-Value & Pooled prevalence, $\%(95 \% \mathrm{Cl})$ \\
\hline \multicolumn{5}{|l|}{ CLINICAL VARIABLES } \\
\hline Disease duration & & & 0.57 & \\
\hline$<10$ years & 6 & $6.5(4.0-10.4)$ & & 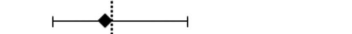 \\
\hline $10-15$ years & 28 & $6.0(4.9-7.4)$ & & $\mapsto$ \\
\hline $15-20$ years & 11 & $7.1(5.0-9.9)$ & & $\longmapsto$ \\
\hline$>20$ years & 15 & $7.0(5.0-9.8)$ & & $\longmapsto$ \\
\hline \% women & & & 0.17 & \\
\hline$<10 \%$ & 2 & $7.0(3.4-13.9)$ & & $\longmapsto$ \\
\hline $10-20 \%$ & 13 & $4.9(3.6-6.8)$ & & $\longmapsto$ \\
\hline $20-30 \%$ & 26 & $6.6(5.7-8.1)$ & & \\
\hline$>30 \%$ & 24 & $7.7(6.2-9.6)$ & & $\bullet$ \\
\hline \% HLA-B27+ & & & 0.68 & \\
\hline$<70 \%$ & 3 & $7.9(4.0-15.1)$ & & $\longmapsto$ \\
\hline $70-80 \%$ & 7 & $5.7(3.7-8.6)$ & & $\longmapsto$ \\
\hline $80-90 \%$ & 22 & $7.4(5.8-9.4)$ & & 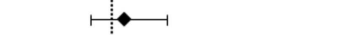 \\
\hline$>90 \%$ & 11 & $6.4(4.5-9.0)$ & & $\longmapsto \bullet$ \\
\hline Geographic area & & & 0.03 & \\
\hline Europe & 39 & $7.2(6.1-8.5)$ & & \\
\hline North-America & 10 & $7.6(5.5-10.4)$ & & $\longrightarrow$ \\
\hline Latin America & 3 & $9.6(5.4-16.9)$ & & $\mapsto$ \\
\hline Asia & 6 & $2.9(1.9-4.4)$ & & $\mapsto \bullet$ \\
\hline Middle-East & 5 & $7.0(4.0-12.0)$ & & $\longmapsto$ \\
\hline \multicolumn{5}{|l|}{ METHODOLOGICAL VARIABLES } \\
\hline Study design & & & 0.72 & \\
\hline Cross-sectional & 38 & $6.3(5.2-7.5)$ & & $\mapsto$ \\
\hline Prospective cohort & 7 & $7.9(5.3-11.6)$ & & $\bullet$ \\
\hline Retrospective cohort & 10 & $7.4(5.3-10.3)$ & & $\bullet$ \\
\hline Case-control & 2 & $8.8(4.3-17.0)$ & & $\bullet$ \\
\hline Intervention & 12 & $7.0(5.0-9.7)$ & & $\longrightarrow$ \\
\hline Sample size & & & 0.33 & \\
\hline$<500$ patients $(n=51)$ & 51 & $7.2(6.0-8.5)$ & & $\mapsto$ \\
\hline$>500$ patients $(n=18)$ & 18 & $6.2(5.0-7.7)$ & & $\mapsto \bullet$ \\
\hline \multicolumn{5}{|l|}{ Risk of bias criteria* } \\
\hline 1. Sampling frame & & & 0.73 & \\
\hline Outpatients (+) & 43 & $6.6(5.6-7.9)$ & & $\mapsto$ \\
\hline Primary care $(+)$ & 2 & $9.9(4.8-20.6)$ & & $\longmapsto$ \\
\hline Register $(+)$ & 15 & $6.4(4.9-8.2)$ & & $\mapsto$ \\
\hline Patient society (-) & 3 & $7.7(4.5-13.0)$ & & $\longmapsto$ \\
\hline 2. Case definition for AS & & & 0.56 & \\
\hline Mod New York (+) & 54 & $6.8(5.8-7.9)$ & & \\
\hline New York (-) & 6 & $5.6(3.7-8.5)$ & & $\longmapsto$ \\
\hline 3. Random selection of patients & & & 0.70 & \\
\hline Specific in- and exclusion criteria (-) & 50 & $6.7(5.7-7.8)$ & & 1 \\
\hline Random selection of patients (+) & 18 & $7.1(5.5-9.1)$ & & $\longmapsto$ \\
\hline 4. Response rate & & & 0.08 & \\
\hline$\geq 70 \%(+)$ & 11 & $6.5(5.4-7.8)$ & & $\mapsto$ \\
\hline$<70 \%(-)$ & 6 & $8.5(6.7-10.7)$ & & $\bullet$ \\
\hline 5. Case definition for EAM & & & 0.71 & \\
\hline Self-report (-) & 15 & $6.4(4.9-8.4)$ & & $\longmapsto$ \\
\hline Interview (+) & 8 & $6.1(4.1-9.0)$ & & $\longmapsto$ \\
\hline Medical records (+) & 22 & $6.4(5.1-8.0)$ & & $\mapsto$ \\
\hline Screening $(+)$ & 2 & $6.7(2.4-17.6)$ & & 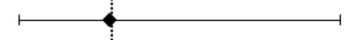 \\
\hline Risk of bias composite score** & & & 0.99 & \\
\hline Low risk of bias (score 4) & 12 & $6.8(4.9-9.3)$ & & $\longmapsto$ \\
\hline Possible risk of bias (score $<4$ ) & 57 & $6.8(5.9-7.9)$ & & 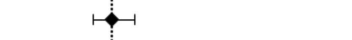 \\
\hline All studies & 69 & $6.8(6.1-7.7)$ & & +1 \\
\hline
\end{tabular}

Figure 4 Prevalence of IBD in patients with ankylosing spondylitis grouped by clinical and methodological characteristics. IBD, inflammatory bowel disease; EAM, extra-articular manifestation. * Individual risk of bias criteria, ' + ' considered as low risk of bias, '-' as high risk of bias. **Sum of individual risk of bias scores, the item on response rate was not considered in the composite score because too many scores were not reported.

the different clinical and methodological characteristics. Only geographical area was associated with heterogeneity in the subgroup analysis $(\mathrm{p}=0.03)$, with the highest prevalence in Latin America (9.6\%) and the lowest in studies from Asia (2.9\%).

\section{Metaregression analysis}

Table 2 shows the results of the univariable and multivariable metaregression analysis of clinical and methodological characteristics exploring the heterogeneity of the reported prevalences. 
Table 2 Univariable and multivariable metaregression analysis of characteristics to explain heterogeneity of prevalence of extra-articular manifestations in patients with AS

\begin{tabular}{|c|c|c|c|c|c|c|c|c|c|c|c|c|}
\hline \multirow[b]{3}{*}{ Variable } & \multicolumn{4}{|l|}{ AAU } & \multicolumn{4}{|l|}{ Psoriasis } & \multicolumn{4}{|l|}{ IBD } \\
\hline & \multicolumn{2}{|l|}{ Univariable } & \multicolumn{2}{|l|}{ Multivariable* } & \multicolumn{2}{|l|}{ Univariable } & \multicolumn{2}{|l|}{ Multivariablet } & \multicolumn{2}{|l|}{ Univariable } & \multicolumn{2}{|l|}{ Multivariable } \\
\hline & $\boldsymbol{\beta}(95 \% \mathrm{Cl})$ & $\mathrm{p}$ Value & $\boldsymbol{\beta}(95 \% \mathrm{Cl})$ & $p$ Value & $\boldsymbol{\beta}(95 \% \mathrm{Cl})$ & $\mathrm{p}$ Value & $\boldsymbol{\beta}(95 \% \mathrm{Cl})$ & $\mathrm{p}$ Value & $\boldsymbol{\beta}(95 \% \mathrm{Cl})$ & $\mathrm{p}$ Value & $\boldsymbol{\beta}(95 \% \mathrm{Cl})$ & $\mathrm{p}$ Value \\
\hline \multicolumn{13}{|l|}{ Clinical variables } \\
\hline Disease duration, year & 0.06 (0.05 to 0.08$)$ & $<0.01$ & 0.05 (0.03 to 0.06$)$ & $<0.01$ & $0.02(-0.01$ to 0.04$)$ & 0.14 & - & - & 0.02 (0.00 to 0.04 ) & 0.04 & - & - \\
\hline$\%$ women & 0.01 (0.00 to 0.02 ) & 0.18 & - & - & $-0.01(-0.03$ to 0.02$)$ & 0.64 & - & - & 0.02 (0.01 to 0.03 ) & 0.02 & 0.02 (0.00 to 0.03$)$ & 0.05 \\
\hline$\%$ HLA-B27+patients & $0.01(0.00$ to 0.02$)$ & 0.09 & - & - & $0.00(-0.01$ to 0.02$)$ & 0.58 & - & - & $-0.01(-0.02$ to 0.01$)$ & 0.28 & - & - \\
\hline \multicolumn{13}{|l|}{ Geographical area } \\
\hline Europe & Reference & - & Reference & - & Reference & - & Reference & - & Reference & - & Reference & - \\
\hline North America & $0.27(-0.04$ to 0.58$)$ & 0.08 & $0.17(-0.10$ to 0.44$)$ & 0.21 & $-0.44(-0.85$ to -0.03$)$ & 0.04 & $-0.44(-0.85$ to -0.03$)$ & 0.04 & $0.05(-0.32$ to 0.43$)$ & 0.78 & $0.15(-0.22$ to 0.51$)$ & 0.43 \\
\hline Latin America & $-0.51(-0.85$ to -0.15$)$ & $<0.01$ & $-0.65(-1.00$ to -0.30$)$ & $<0.01$ & $-0.06(-1.33$ to 1.21$)$ & 0.93 & $-0.06(-1.33$ to 1.21$)$ & 0.93 & $0.32(-0.32$ to 0.96$)$ & 0.33 & 0.40 (-0.23 to 1.03$)$ & 0.21 \\
\hline Asia & $-0.42(-0.64$ to -0.19$)$ & $<0.01$ & $-0.22(-0.45$ to -0.00$)$ & 0.05 & $-1.33(-2.09$ to -0.57$)$ & $<0.01$ & $-1.33(-2.09$ to -0.57$)$ & $<0.01$ & $-0.96(-1.44$ to -0.49$)$ & $<0.01$ & $-0.80(-1.29$ to -0.32$)$ & $<0.01$ \\
\hline Middle East & $-0.64(-0.97$ to -0.30$)$ & $<0.01$ & $-0.38(-0.67$ to -0.09$)$ & 0.01 & $-1.03(-1.73$ to -0.32$)$ & $<0.01$ & $-1.03(-1.73$ to -0.32$)$ & $<0.01$ & $-0.03(-0.64$ to 0.58$)$ & 0.92 & $-0.01(-0.61$ to 0.59$)$ & 0.98 \\
\hline Australia & $-0.76(-1.90$ to 0.38$)$ & 0.19 & $-\S$ & - & - & - & - & - & - & - & - & - \\
\hline \multicolumn{13}{|l|}{ Methodological variables } \\
\hline \multicolumn{13}{|l|}{ Risk of bias criteria } \\
\hline Sampling frameף & $-0.22(-0.47$ to 0.03$)$ & 0.09 & - & - & $-0.37(-0.80$ to 0.05$)$ & 0.08 & - & - & $-0.21(-0.64$ to 0.23$)$ & 0.35 & - & - \\
\hline Case definition for ASף & $-0.08(-0.31$ to 0.14$)$ & 0.48 & - & - & $-0.20(-0.58$ to 0.19$)$ & 0.32 & - & - & $0.02(-0.31$ to 0.36$)$ & 0.89 & - & - \\
\hline Random selectionๆ & $-0.22(-0.46$ to 0.02$)$ & 0.07 & $-0.24(-0.43$ to -0.04$)$ & 0.02 & $-0.12(-0.50$ to 0.25$)$ & 0.53 & - & - & $0.06(-0.26$ to 0.38$)$ & 0.70 & - & - \\
\hline Case definition for EAMף & $0.00(-0.20$ to 0.20$)$ & 0.99 & - & - & $-0.08(-0.43$ to 0.26$)$ & 0.64 & - & - & $-0.26(-0.57$ to 0.05$)$ & 0.10 & - & - \\
\hline \multicolumn{13}{|c|}{ Study design } \\
\hline Cross-sectional & Reference & - & - & - & Reference & - & - & - & Reference & - & - & - \\
\hline Prospective cohort & $0.27(-0.12$ to 0.66$)$ & 0.11 & - & - & $0.27(-0.31$ to 0.85$)$ & 0.37 & - & - & $0.25(-0.23$ to 0.72$)$ & 0.31 & - & - \\
\hline Retrospective cohort & $-0.20(-0.45$ to 0.05$)$ & 0.12 & - & - & $0.46(0.02$ to 0.90$)$ & 0.04 & - & - & $0.18(-0.23$ to 0.59$)$ & 0.11 & - & - \\
\hline Case-control study & $0.75(-0.35$ to 1.86$)$ & 0.19 & - & - & $0.02(-0.81$ to 0.84$)$ & 0.96 & - & - & 0.36 (-0.42 to 1.14$)$ & 0.37 & - & - \\
\hline Intervention study & $0.20(-0.10$ to 0.49$)$ & 0.19 & - & - & $0.48(0.07$ to 0.89$)$ & 0.02 & - & - & $0.11(-0.29$ to 0.52$)$ & 0.58 & - & - \\
\hline Sample size & $0.00(0.00$ to 0.00$)$ & 0.13 & - & - & $0.00(0.00$ to 0.00$)$ & 0.48 & - & - & $0.00(0.00$ to 0.00$)$ & 0.62 & - & - \\
\hline
\end{tabular}

Parameters with a $\mathrm{p}$ value $>0.30$ in univariable analysis were entered in the multivariable model and removed in order of significance if $p>0.05$ (backward procedure).

*Multivariable model of AAU was based on 119 studies.

† Multivariable model of psoriasis was based on 56 studies.

F Multivariable model of IBD was based on 65 studies.

SThe study on prevalence of uveitis from Australia was excluded from multivariable analysis because data on disease duration were missing.

AAU, acute anterior uveitis; AS, ankylosing spondylitis; EAM, extra-articular manifestation; HLA-B27,Human Leukocyte Antigen-B27; IBD, inflammatory bowel disease. 
No interaction was found between the variables. All VIF statistics were well below the recommended cut-off value of 10 .

\section{Acute anterior uveitis}

In the final multivariable metaregression model on the prevalence of AAU in AS, the clinical characteristics disease duration ( $\beta 0.05,95 \%$ CI 0.03 to 0.06$)$ and geographical area $(\beta-0.64$, $95 \%$ CI -1.00 to -0.28 in studies in the Middle East compared with studies from Europe) remained significantly associated with the prevalence of AAU. Random selection of patients, was the only 'risk of bias' criterion which was significantly associated with prevalence of AAU ( $\beta-0.24,95 \%$ CI -0.43 to -0.04$)$. The regression model explained $44.9 \%$ of the total heterogeneity.

\section{Psoriasis}

In the multivariable metaregression model on the prevalence of psoriasis in AS, only geographical area was significantly associated with the prevalence of psoriasis, with lower prevalence estimates in studies from Asia $(\beta-1.33$, 95\% CI -2.09 to $-0.57)$ and the Middle East $(\beta-1.03,95 \% \mathrm{CI}-1.73$ to -0.32$)$ compared to studies from Europe. The regression model explained $27.2 \%$ of the total heterogeneity.

\section{Inflammatory bowel disease}

In the multivariable meta-regression model on the prevalence of IBD, geographical area (lower in studies from Asia ( $\beta-0.80,95 \%$ CI -1.29 to -0.32 ) compared to Europe) and the percentage of women included in the studies ( $\beta 0.02,95 \%$ CI 0.00 to 0.03 ) were significantly associated with the prevalence of IBD. The regression model explained $25.7 \%$ of the total heterogeneity.

\section{DISCUSSION}

The present review confirmed high prevalences of EAMs in patients with AS. The pooled prevalence of AAU in patients with AS was $25.8 \%$, whereas, a lifetime cumulative incidence of $\mathrm{AAU}$ in the general population is reported to be $0.2 \%$ in HLA-B27-negative and 1\% in HLA-B27-positive subjects. ${ }^{17}$ The pooled prevalence of psoriasis in AS was $9.3 \%$ and for IBD $6.8 \%$, both are considerably higher than general population estimates which vary from $0.3 \%$ to $2.5 \%$ for psoriasis, and from $0.01 \%$ to $0.5 \%$ for IBD. ${ }^{18} 19$

This study is the first using a systematic approach with meta-analysis to estimate the prevalence of AAU, psoriasis and IBD in patients with AS. Zeboulon et al performed an SLR on the prevalence of AAU and reported a (crude) prevalence of $33.2 \%$ (mean disease duration of 17 years), which increased with disease duration and was higher in HLA-B27-positive patients. The prevalence is comparable to our study, although we found a lower prevalence (20\%), when limiting the analysis to high-quality studies. While we confirmed a trend of higher prevalences in studies with a higher percentage of HLA-B27-positive patients, this was not significant in our multivariable analyses. SLRs on the prevalence of psoriasis and IBD in AS are lacking. The present findings are in the lower range of the general estimates of $5-10 \%$ and $10-25 \%$ for IBD and psoriasis, respectively.

A metaregression analysis was performed to explore the methodological and clinical heterogeneity among the studies. Disease duration and geographical area were the most striking contributors to heterogeneity found. Specifically, the prevalence of AAU was associated with disease duration, and ranged from $17.4 \%$ among studies in patients with a mean disease duration of $<10$ years to $38.5 \%$ among studies in patients with a mean disease duration of $>20$ years. This association with disease duration was neither found for psoriasis nor for IBD. This suggests, but does not prove, that psoriasis and IBD may already be present before the onset or before diagnosis of AS. It should be noted, however, that studies including patients with a short disease duration ( $<10$ years) were under-represented in the present review. Because the EAMs are now part of the classification criteria for axSpA ${ }^{20}$ and, moreover, are considered as contributory to a diagnosis of axSpA, ${ }^{3}$ the relationship between the onset of AS and onset of EAMs needs more attention in future studies.

Interestingly, variation in prevalence of EAMs was found across different geographical areas. Genetic factors may explain part of the variation. It is known that AAU is more prevalent in HLA-B27-positive patients compared with HLA-B27-negative patients. ${ }^{21}$ As there is a geographical difference in the percentage of patients with AS who possess HLA-B27, ${ }^{22} 23$ it could be expected that part of the geographical variation in the prevalence of EAM is due to the prevalence of HLA-B27. In the present study, however, most differences between geographical areas remained significant when controlling for HLA-B27 in the multivariable regression, suggesting that other (epi)genetic factors, are likely to play a role. It should be reminded that the number of studies and patients included from Africa, Latin America and Australia were limited, and that in metaregressions, associations with HLA-B27 and EAM are explored at the study level and not at the individual patient level.

A lower risk of bias based on the composite score tended to provide lower estimates of the prevalence of AAU and psoriasis in univariable analyses. In the multivariable analysis, high risk of bias in random selection of patients contributed independently to higher prevalence rates of AAU. A possible explanation could be that patients in trials were selected based on higher disease activity and that this may coincide with a higher prevalence of AAU. ${ }^{24}$

Some limitations of the present study should be recognised. First, the prevalence of EAMs used in this review was usually not the main objective of the primary studies included, and therefore, not always indexed in the electronic article databases. We applied a broad search strategy without any terms for 'prevalence' or 'epidemiology'. However, despite additional efforts of the authors, probably not all studies reporting EAMs in AS were captured with the search strategy. This was also emphasised by the high number of additional articles that were retrieved by 'snowballing'. Second, despite the fact that all studies were checked for double counting of the same cohort, we cannot fully exclude that subgroups of patients participated in several studies, and therefore, were included twice (or more) in the assessment of the EAM. Third, a large heterogeneity was found, and although this was explored in subgroup and metaregression analyses, these analyses have some limitations. ${ }^{14}$ Results from metaregressions are observational, and therefore, can suffer from bias by confounding, which means that an association identified with one study characteristic may reflect a true association with other correlated characteristics, whether these are known or unknown. Moreover, in some studies, information was lacking on, for example, disease duration. Therefore, analyses could only be based on the studies with available information, potentially biasing the results. Further, patients' characteristics are based on group-averages. Such analyses are difficult to interpret, because the relationship on study level may not be the same as the relationship on patient level. Last, the variability between studies may be lower than the within-studies, which makes it sometimes more difficult to show significant results of true relationships. 
In conclusion, this SLR with meta-analysis summarised the prevalence of AAU, psoriasis and IBD in patients with AS. Awareness of EAMs, among clinicians, is important in view of their role in the diagnostic process, for treatment choices and for health-related quality of life. The prevalence of EAMs among patients with AS is clearly increased compared with the general population. While AAU occurs clearly more frequently in patients with longer disease duration, this was less clear for IBD and psoriasis.

Acknowledgements We acknowledge Louise Falzon, Information Specialist at the Center for Behavioral Cardiovascular Health, Columbia University Medical Center, for assistance with the development of the search strategy; Merdan Saritas, fellow in Rheumatology at the Maastricht University Medical Center, for translations from Turkish language, and Antje van der Zee-Neuen, PhD student at Maastricht University, for translations from German language.

Contributors CS, AvT and AB participated in the design of the study and the data interpretation. CS, JC and $A B$ extracted the data. All authors read and approved the final manuscript.

\section{Competing interests None.}

Provenance and peer review Not commissioned; externally peer reviewed.

\section{REFERENCES}

1 Sieper J, Braun J, Rudwaleit M, et al. Ankylosing spondylitis: an overview. Ann Rheum Dis 2002;61:8-18.

2 Elewaut D, Matucci-Cerinic M. Treatment of ankylosing spondylitis and extra-articular manifestations in everyday rheumatology practice. Rheumatology (Oxford) 2009;48:1029-35.

3 Rudwaleit M, van der Heijde D, Khan MA, et al. How to diagnose axial spondyloarthritis early. Ann Rheum Dis 2004;63:535-43.

4 de Groot V, Beckerman H, Lankhorst GJ, et al. How to measure comorbidity. a critical review of available methods. J Clin Epidemiol 2003;56:221-9.

5 Brophy S, Pavy S, Lewis P, et al. Inflammatory eye, skin, and bowel disease in spondyloarthritis: genetic, phenotypic, and environmental factors. I Rheumatol 2001:28:2667-73.

6 Chorus AM, Miedema HS, Boonen A, et al. Quality of life and work in patients with rheumatoid arthritis and ankylosing spondylitis of working age. Ann Rheum Dis 2003;62:1178-84.

7 Robertson LP, Davis MJ. A longitudinal study of disease activity and functional status in a hospital cohort of patients with ankylosing spondylitis. Rheumatology (Oxford) 2004;43:1565-8.
8 Baeten D, Breban M, Lories R, et al. Are spondylarthritides related but distinct conditions or a single disease with a heterogeneous phenotype? Arthritis Rheum 2013;65:12-20.

9 Zeboulon N, Dougados M, Gossec L. Prevalence and characteristics of uveitis in the spondyloarthropathies: a systematic literature review. Ann Rheum Dis 2008;67:955-9.

10 Shamliyan TA, Kane RL, Ansari MT, et al. Development quality criteria to evaluate nontherapeutic studies of incidence, prevalence, or risk factors of chronic diseases: pilot study of new checklists. J Clin Epidemiol 2011;64:637-57.

11 Higgins JP, Thompson SG. Quantifying heterogeneity in a meta-analysis. Stat Med 2002;21:1539-58.

12 Higgins JP, Thompson SG, Deeks JJ, et al. Measuring inconsistency in meta-analyses. Bmj 2003;327:557-60.

13 Lipsey M, Wilson D. Practical meta-analysis. California: Sage publications, 2001.

14 Thompson SG, Higgins JP. How should meta-regression analyses be undertaken and interpreted? Stat Med 2002;21:1559-73.

15 Myers RH. Classical and modern regression with applications. Boston, MA: PWS-Kent, 1990.

16 Wilson D. METAREG for SPSS/Win 6.1 or higher MACRO. 2001. http://mason.gmu. edu/ dwilsonb/ma.html

17 Linssen A, Rothova A, Valkenburg HA, et al. The lifetime cumulative incidence of acute anterior uveitis in a normal population and its relation to ankylosing spondylitis and histocompatibility antigen HLA-B27. Invest Ophthalmol Vis Sci 1991;32:2568-78.

18 Plunkett A, Marks R. A review of the epidemiology of psoriasis vulgaris in the community. Australas I Dermatol 1998;39:225-32.

19 Molodecky NA, Soon IS, Rabi DM, et al. Increasing incidence and prevalence of the inflammatory bowel diseases with time, based on systematic review. Gastroenterology 2012;142:46-54 e42; quiz e30.

20 Rudwaleit M, van der Heijde D, Landewe R, et al. The development of Assessment of SpondyloArthritis international Society classification criteria for axial spondyloarthritis (part II): validation and final selection. Ann Rheum Dis 2009;68:777-83.

21 Feldtkeller E, Khan MA, van der Heijde D, et al. Age at disease onset and diagnosis delay in HLA-B27 negative vs. positive patients with ankylosing spondylitis. Rheumatology International 2003;23:61-6.

22 Stolwijk C, Boonen A, van Tubergen A, et al. Epidemiology of spondyloarthritis. Rheum Dis Clin North Am 2012;38:441-76.

23 Abdelrahman MH, Mahdy S, Khanjar IA, et al. Prevalence of HLA-B27 in Patients with Ankylosing Spondylitis in Qatar. Int I Rheumatol 2012;2012: 860213.

24 Chen $\mathrm{CH}$, Lin KC, Chen HA, et al. Association of acute anterior uveitis with disease activity, functional ability and physical mobility in patients with ankylosing spondylitis: a cross-sectional study of Chinese patients in Taiwan. Clinical rheumatology 2007;26:953-7. 